\title{
Design of CIC based decimation filter structure using FPGA for WiMAX applications
}

\author{
V. Jayaprakasan ${ }^{1 \mathrm{a})}$, S. Vijayakumar ${ }^{2 \mathrm{~b})}$, and \\ Pandya Vyomal Naishadhkumar ${ }^{1 \mathrm{c})}$ \\ ${ }^{1}$ Department of ECE, Sreenidhi Institute of Science and Technology (Autonomous) \\ Hyderabad, Telengana-501301, India \\ ${ }^{2}$ Department of ECE, Sreenivasa Institute of Technology and Management Studies \\ Autonomous, Chittoor, Andhra Pradesh-517127, India \\ a) jayaprakasanv@sreenidhi.edu.in \\ b)vijaysuresh1975@yahoo.com \\ c)vyomalpandya@sreenidhi.edu.in
}

\begin{abstract}
This paper focuses on the efficient design and FPGA realization of CIC based decimation filter structure for WiMAX application. This structure reduces the sampling rate at each section and lower the power consumption in each section with improved magnitude response. The magnitude responses and device utilization of the filter with different combinations considering different stages are estimated and compared with the existing structures. MATLAB Simulink environment is used for design and Xilinx Virtex-V XC5VLX110T-3ff1136 FPGA is utilized for implementation. It is observed from the results that the passband droop and stopband characteristics of this filter structure are improved when the decimation factor of first section is less compared to second section $(\mathrm{M} 1<\mathrm{M} 2)$. On the other hand power consumption of the filter is less when the decimation factor of first section is high compared with second section (M1 > M2). The results show that the passband droop improvement is about $38 \%$ and stopband attenuation improvement is of $33 \%$ for the decimation factor $\mathrm{M}=8$.
\end{abstract}

Keywords: CIC filter, decimation, FPGA, WiMAX, zeros-distribution Classification: Microwave and millimeter-wave devices, circuits, and modules

\section{References}

[1] M. Laddomada: "Generalized comb decimation filters for $\Sigma \Delta \mathrm{A} / \mathrm{D}$ converters: Analysis and design," IEEE Trans. Circuits Syst. I, Reg. Papers 54 (2007) 994 (DOI: 10.1109/TCSI.2007.895528).

[2] S. Vijayakumar and R. Korah: "Circuit level, $32 \mathrm{~nm}$, 1-bit MOSSI-ULP adder: Power, PDP and area efficient base cell for unsigned multiplier," IEICE Electron. Express 11 (2014) 20140109 (DOI: 10.1587/elex.11.20140109). 
[3] M. Laddomada: "Comb-based decimation filters for $\Sigma \Delta$ A/D converters: Novel schemes and comparisons," IEEE Trans. Signal Process. 55 (2007) 1769 (DOI: 10.1109/TSP.2006.890822).

[4] G. J. Dolecek and F. Harris: "Design of CIC compensator filter in a digital IF receiver," IEEE International Symp. Comm. and Info. Tech. (2008) 638 (DOI: 10.1109/ISCIT.2008.4700271).

[5] G. J. Dolecek and F. Harris: "On design of two-stage CIC compensation filter," IEEE International Symposium - ISIE (2009) 903 (DOI: 10.1109/ISIE.2009. 5213597).

[6] G. J. Dolecek and S. K. Mitra: "Two-stage CIC-based decimator with improved Characteristics," IET Signal Process. 4 (2010) 22 (DOI: 10.1049/iet-spr.2008. 0160).

[7] A. Fernandez-Vazquez and G. J. Dolecek: "Maximally flat CIC compensation filter: Design and multiplierless implementation," IEEE Trans. Circuits Syst. II, Exp. Briefs 59 (2012) 113 (DOI: 10.1109/TCSII.2011.2180093).

[8] C. Jeong, et al:: "Double-sharpened decimation filter employing a pre-droop compensator for multi-standard wireless applications," ETRI 33 (2011) 169 (DOI: 10.4218/etrij.11.0110.0272).

[9] T. K. Shahana, et al:: "GUI based decimation filter design tool for multistandard wireless transceivers," IEEE (2007) (DOI: 10.1109/EDSSC.2007. 4450130).

[10] L. Milic: Multirate Filtering for Digital Signal Processing: MATLAB Applications (Information Science Reference, NY, USA, 2009).

\section{Introduction}

The decimation filter finds wide application in both analog and digital systems for the purpose of data rate conversion as well as filtering. It has been widely used in audio, speech processing, radar systems and communication systems. CIC (Cascaded-Integrator-Comb) filter structure is one of the filter structure which requires less coefficients multipliers are not needed. Though the hardware requirement is reduced, the frequency response is not improved. This is the motivation for the present work to focus on design and realization of efficient CIC based decimation filter structure with improved magnitude response.

The design and implementation of different decimation filter structures of CIC with various techniques have been reported in the past few decades by many researchers $[1,2,3,4,5,6,7,8,9,10]$. In this paper is the design and implement the multi-section CIC based decimation filter structure for WiMAX application is proposed which can operate at a lower sampling rate to achieve better performance than the conventional comb filter structure.

This paper is organized as follows. Section 2 discusses the modified CIC Decimation filter with its magnitude responses. Section 3 presents the proposed CIC based decimation filter structure in detail. The FPGA implementation and results are discussed in section 4. Conclusion is given in section 5 .

\section{Modified CIC decimation filter}

In order to improve the magnitude characteristics, the CIC decimation filter can be constructed using two sections with different number of stages, resulting in a 
modified CIC filter as shown in Fig. 1. In modified CIC filter structure, the decimation factor $M$ is divided in to two $(M=M 1 M 2)$.

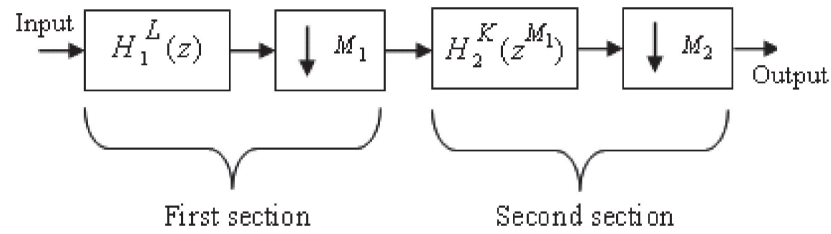

Fig. 1. Modified CIC decimation filter

The transfer function of modified CIC filter can be written as

$$
H_{m}(z)=\left[H_{1}{ }^{L}(z)\right]\left[H_{2}{ }^{K}\left(z^{M_{1}}\right)\right]
$$

where,

$$
\begin{aligned}
H_{1}(z) & =\frac{1}{M_{1}}\left(\frac{1-z^{-M_{1}}}{1-z^{-1}}\right) \\
H_{2}\left(z^{M_{1}}\right) & =\frac{1}{M_{2}}\left(\frac{1-z^{-M_{1} M_{2}}}{1-z^{-M_{1}}}\right)
\end{aligned}
$$

Here $H_{1}{ }^{L}(z)$ and $H_{2}{ }^{K}\left(z^{M_{1}}\right)$ are CIC filters, with decimation factor M1 and M2 respectively. The first CIC filter section of decimation factor M1 works at the high input rate and the second section are with decimation factor M2 which works at the lower rate.

The magnitude response can be increased by introducing the sharpening technique [4] in the second section. The transfer function of sharpened CIC filter can be written as

$$
H_{s h}(z)=3[H(z)]^{2 K}-2[H(z)]^{3 K}
$$

Hence the transfer function of modified sharpened CIC filter [8] can be rewritten as

$$
H_{m, s h}(z)=\left[H_{1}(z)\right]^{L}\left[3 H_{2}\left(z^{M_{1}}\right)^{2 K}-2 H_{2}\left(z^{M_{1}}\right)^{3 K}\right]
$$

\section{Proposed CIC based decimation filter}

In order to improve the magnitude characteristics, the proposed CIC decimation filter can be constructed using two sections with different number of stages with sharpening and zeros distribution techniques, as shown in Fig. 2.

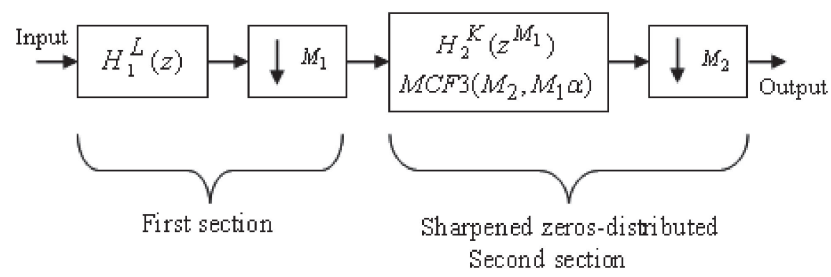

Fig. 2. Proposed structure (without compensation)

The performance of CIC decimation filter can be improved by applying zero distribution technique [3] in such a way as to guarantee the best stopband attenuation around the folding bands. This technique is applied to the second section of modified sharpened filter which distributes the nulls in the sharpened 
section. Modified Comb Filter (MCF) with distributed zeroes $[4,5]$ has better distribution of nulls than multistage sharpened CIC filter in the stopband attenuation in the aliasing band. The transfer function of MCF3 $(\mathrm{M}, \alpha)$ can be represented by

$$
H_{M C F 3}(z)=H_{C I C}(z) H_{\text {rot }+}(z) H_{\text {rot }-}(z)
$$

where, $H_{r o t+}(z)$ is the transfer function of positive distribution

$H_{\text {rot- }}(z)$ is the transfer function of negative distribution

$$
\begin{aligned}
& H_{r o t+}(z)=\frac{1}{M}\left(\frac{1-e^{j M \alpha} z^{-M}}{1-e^{j \alpha} z^{-1}}\right) \\
& H_{r o t-}(z)=\frac{1}{M}\left(\frac{1-e^{-j M \alpha} z^{-M}}{1-e^{-j \alpha} z^{-1}}\right)
\end{aligned}
$$

Multiplying "(7)" and "(8)" together, the transfer function of distribution function can be expressed as

$$
H_{r o t}(z)=H_{r o t+}(z) H_{r o t-}(z)=\frac{1}{M^{2}}\left(\frac{1-2 \cos (\alpha M) z^{-M}+z^{-2 M}}{1-2 \cos (\alpha) z^{-1}+z^{-2}}\right)
$$

The zeros distribution with sharpening technique in the second section is expected to improve the magnitude response compared to existing CIC filter structures. This proposed structure provides better stop band attenuation compared with the existing methods, but the attenuation at pass band edge increased significantly. This can be decreased by the use of compensator. The decimated output obtained from the second section is given to the compensator to decrease the attenuation at pass band edge. The pass band droop will depend on the sine compensator and the proposed structure with compensator is shown in Fig. 3. The generalized transfer function of compensator is given as,

$$
G_{c o m}\left(z^{M}\right)=A\left[1+B z^{-M}+z^{-2 M}\right]
$$

Where $A=-2^{-(b+2)} ; B=-\left(2^{-(b+2)}+2\right)$.

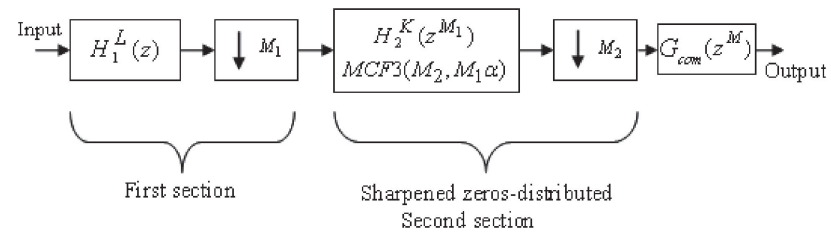

Fig. 3. Proposed structure (with compensation)

The compensation filter parameter " $\mathrm{b}$ " depends on the value of $\mathrm{K}$, not on the decimation factor M. For the given value of " $b$ " and $\mathrm{K}$, the values of decimation factors are not expected to affect the worst case alias rejection. The block diagram of proposed CIC filter with compensator is shown in Fig. 4. The first section is comb decimator with decimation factor $\mathrm{M}_{1}$ which can be realized in either recursive or non-recursive scheme. As a result second section is moved to a lower rate which is $\mathrm{M}_{1}$ times lower than the input rate. The compensation filter plays an important role for compensation of the pass band attenuation introduced by the second section. The transfer function of the proposed CIC decimation filter with compensator can be written as, 


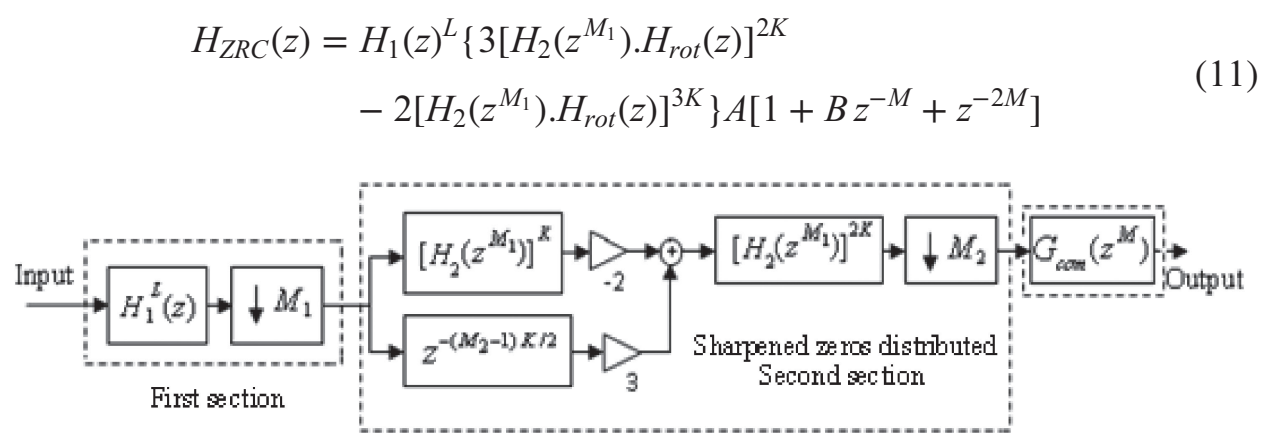

Fig. 4. Block diagram of CIC decimation filter structure

\section{FPGA implementation and results}

The CIC based decimation filter structure with sharpening, zeros distribution and compensation techniques are developed for decimation factor $\mathrm{M}=8$. The device utilization, power consumption, pass band droop and stop band alias rejection of CIC based decimation filter structure for the decimation factor $\mathrm{M}=8$ with $\mathrm{K}=1$ and $\mathrm{L}=2$ are computed and compared with various methods outlined in $[1,7]$ using MATLAB and Xilinx System Generator. The performance of the CIC decimation filter structure with various parameters are given in Table I. Fig. 5 shows the model of the proposed CIC filter with $M=8$.

Table I. Filter design parameters considered for analysis

\begin{tabular}{|l|l|}
\hline Parameters & Values for WiMAX \\
\hline Compensation factor, $\mathrm{b}$ & 6 for $\mathrm{K}=1$ \\
\hline Filter Quality factor, $q$ & 0.78 \\
\hline Maximum signal frequency, $f_{m}$ & 0.02 (Normalized) \\
\hline Over Sampling Rate & 8 \\
\hline Input sampling frequency $\mathrm{F}_{\mathrm{s}}(\mathrm{MHz})$ & 133.632 \\
\hline Pass band Edge $(\mathrm{MHz})$ & 8 \\
\hline Stop band Edge $(\mathrm{MHz})$ & 10 \\
\hline Pass band ripple $(\mathrm{dB})$ & 0.5 \\
\hline Stop band attenuation $(\mathrm{dB})$ & 39 \\
\hline
\end{tabular}

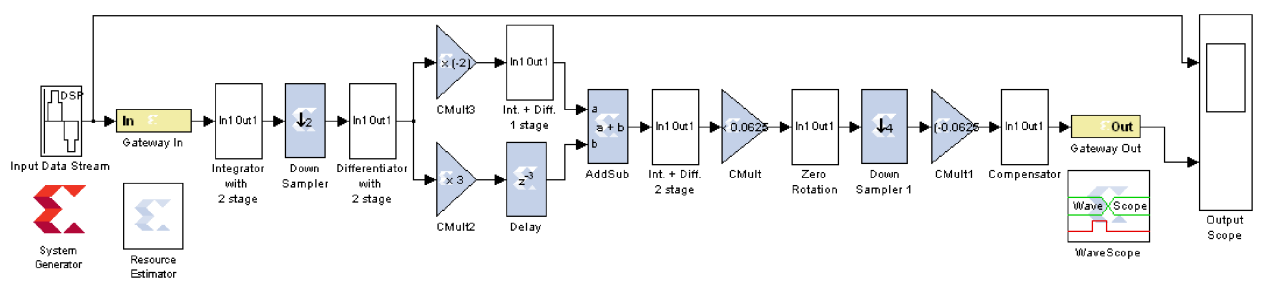

Fig. 5. FPGA realization of proposed CIC filter structure for $M=8$ with $\mathrm{K}=1$ and $\mathrm{L}=2$

The magnitude response of the decimation filter without and with compensator for $\mathrm{M}=8$ considering $\mathrm{K}=1$ and $\mathrm{L}=2$ is shown in Fig. 6. From the overall magnitude response of CIC filter without and with compensator as in Fig. 6 with pass band droop is improved and the stop band alias rejection is reduced compared to the structures reported earlier. 


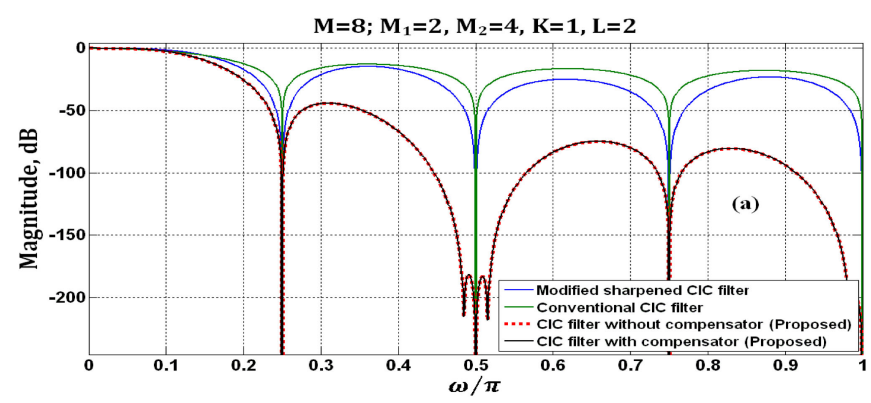

Fig. 6. Magnitude responses plots for $M=8, M_{1}=2, M_{2}=4$ with $\mathrm{K}=1$ and $\mathrm{L}=2$.

Table II. Overall comparisons of passband droop and stopband alias rejection with device utilization and power consumption for $\mathrm{M}=8 ; \mathrm{M}_{1}=2 ; \mathrm{M}_{2}=4$ with $\mathrm{K}=1$ and $\mathrm{L}=2$

\begin{tabular}{|c|c|c|c|c|c|c|c|c|c|c|c|}
\hline $\begin{array}{c}\text { Filter } \\
\text { Structure }\end{array}$ & $\mathbf{M}$ & $M_{1}$ & $\mathbf{M}_{2}$ & $\mathbf{K}$ & $\mathbf{L}$ & $\begin{array}{c}\text { Number } \\
\text { of } \\
\text { Slice } \\
\text { Registers }\end{array}$ & $\begin{array}{c}\text { Number } \\
\text { of } \\
\text { Slice } \\
\text { LUTs }\end{array}$ & 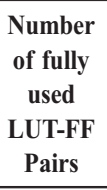 & $\begin{array}{c}\text { Power } \\
\text { Consumption } \\
\text { in Watts }\end{array}$ & $\begin{array}{l}\text { Passband } \\
\text { Droop } \\
\text { (dB) }\end{array}$ & $\begin{array}{l}\text { Stopband } \\
\text { Alias } \\
\text { Rejection } \\
\text { (dB) }\end{array}$ \\
\hline $\begin{array}{l}\text { Modified } \\
\text { Sharpened } \\
\text { CIC [8] }\end{array}$ & 8 & 2 & 4 & 1 & 2 & 320 & 550 & 627 & 0.036 & -0.00616 & -37.50 \\
\hline $\begin{array}{l}\text { Conventional } \\
\text { CIC [1] }\end{array}$ & 8 & - & - & 1 & & 75 & 64 & 115 & 0.040 & -0.055 & -23.28 \\
\hline $\begin{array}{l}\text { Proposed } \\
\text { CIC without } \\
\text { compensator }\end{array}$ & 8 & 2 & 4 & 1 & 2 & 418 & 764 & 883 & 0.041 & -0.00888 & -50.125 \\
\hline $\begin{array}{l}\text { Proposed } \\
\text { CIC with } \\
\text { compensator }\end{array}$ & 8 & 2 & 4 & 1 & 2 & 467 & 949 & 1045 & 0.064 & -0.00378 & -50.12 \\
\hline \multicolumn{10}{|c|}{ Passband Droop and Stopband Attenuation Improvement in Percentage } & $38 \%$ & $33 \%$ \\
\hline
\end{tabular}

It is clear from the Table II that the CIC decimation filter with compensator improves the passband droop and stopband alias rejection performance compared to the existing and conventional CIC filter structures. It meets the required WiMAX specification. Compared to the existing structures, the proposed CIC decimation filter structure gives 38\% improvement in passband droop and 33\% stopband performance for decimation factor $\mathrm{M}=8$.

\section{Conclusion}

The sharpened zero rotated CIC decimation filter was developed and implemented with the help of Xilinx-Virtex-V FPGA for decimation factor 8. The evaluation shows that the passband droop and the stopband attenuation of the proposed CIC filter structure are improved compared with the existing filter structures. The droop decreases for higher stages. It is observed that the passband droop and stopband characteristics of this filter is improved when the decimation factor of first section is less compared to second section $\left(\mathrm{M}_{1}<\mathrm{M}_{2}\right)$. The power consumption of the filter is less when the decimation of first section is high compared with second section $\left(\mathrm{M}_{1}>\mathrm{M}_{2}\right)$. It can be WiMAX with factor $\mathrm{M}=8$. 